\title{
Dimensional Evolution of Intelligent Cars Human-Machine Interface considering Take-Over Performance and Drivers' Perception on Urban Roads
}

\author{
Hao Yang, ${ }^{1,2}$ Yueran Wang $\mathbb{D}^{3}{ }^{3}$ and Ruoyu Jia ${ }^{1}$ \\ ${ }^{1}$ College of Mechanical and Material Engineering, North China University of Technology, Beijing, China \\ ${ }^{2}$ Department of Industrial Engineering, Tsinghua University, Beijing, China \\ ${ }^{3}$ School of International Art Education, Tianjin Academy of Fine Arts, Tianjin, China
}

Correspondence should be addressed to Yueran Wang; tafa_wyr@163.com

Received 11 July 2020; Revised 17 September 2020; Accepted 18 September 2020; Published 1 October 2020

Academic Editor: Jun Yang

Copyright (c) 2020 Hao Yang et al. This is an open access article distributed under the Creative Commons Attribution License, which permits unrestricted use, distribution, and reproduction in any medium, provided the original work is properly cited.

The study analyzed the drivers' take-over behaviors in intelligent cars when driving on urban roads and tried to find reasonable dimensions of the human-machine interface. Firstly, the main driving assistance functions in the process of take-over were analyzed based on the entropy theory, and the weight values of each function for the consumer's purchase intention were calculated. Secondly, we explored the perceived comfortable dimensions of the interactive components under typical interaction modes. By means of experiments using a within-subjects design, the initial population of the evolutionary computation was obtained. The evolutionary mechanism of dimensions driven by users' perception was constructed with a genetic algorithm. After debugging the parameters of the model, we verified the rationality of the model and evolved appropriate dimensions. Finally, the validity of the evolved dimensions was proved by a controlled experiment and paired-sample $t$-test. The results indicated that the completion time of most take-over tasks under the HMI with the evolved dimensions was significantly shorter, which ensured the HMI could be more conducive to the take-over quality and traffic efficiency.

\section{Introduction}

The intelligent vehicle is a comprehensive system which integrates environmental perception, planning, multilevel driving assistance, and other functions. Due to the complexity in intelligent vehicles brought about by technologies in fields of computer, modern sensing, information fusion, communication, artificial intelligence, and automatic control, a good human-vehicle interaction interface is important in order to improve the safety and comfort of driving [1]. With the growth of car ownership and the shortage of road resources, the complexity of urban traffic environment is also increasing [2]. The interaction performance and comfort degree generated by the driving assistant system (DAS) would bring about driver pressure and distraction, which is unfavorable to the sustainable development of traffic efficiency and road environment $[3,4]$. According to the automated driving standard J3016 [5], most of the current automated driving vehicles are at level 2-level 3. Because this level of intelligent system requires the driver to take over at any time, the core of interaction design is human-vehicle codriving. Therefore, the degree of technology acceptance by users must be fully taken into consideration, which is of significance for a safe driving and sustainable urban traffic.

Driving always puts a driver in a nervous state [3]. Therefore, it is necessary to ensure that the driver can easily control the steering wheel, buttons, combined switch paddles, and other components while the body is not drastically changed. This can help meet the physiological requirements of drivers and reduce fatigue to ensure convenient, rapid, and effective operation. This paper focuses on drivers' hand extension problems in take-over tasks under human-computer codriving (HCCD) and looks for an optimal dimension of the human-machine interface (HMI) based on 
perceived comfortable dimensions (PCD) of the core interactive components.

Driving movements are not completed independently by one part of the body, but they are coordinated by multiple parts with coherence. Therefore, the traditional static measurement is difficult to obtain effective size data. When designing the dimension and layout of the HMI, it is useful to set the sizes based on the driver's perception characteristics and comfort degree. Because a user's perception of a product performance may deviate due to the influence of its appearance [6], this paper utilizes multiple measurements to obtain stable PCD data. And the genetic algorithm (GA) was used to evolve the optimal size.

\section{Literature Review}

2.1. Cognitive and Behavioral Problems in the Process of TakeOver. As a research hotspot in the field of transportation, automated driving can greatly improve driving safety and alleviate modern traffic problems such as accidents, fuel emissions, and road congestion [7-9]. Meanwhile, it can promote driving comfort and reduce the impact of the environment [10]. When the automated driving system is out of order or is unable to deal with the traffic conditions, the driver needs to control the vehicle manually so that driving safety can be ensured. The process to arouse the driver's cognition and behavioral control is called take-over. Therefore, as a key link in the safe driving of conditional autonomous vehicles, the take-over process is a focus of HCCD behavior research and interaction design.

After receiving the take-over request, the driver transfers the attention to driving tasks, then obtains the situational awareness, makes the decision, and drives, until the vehicle is fully controlled [11]. If the size and distance of interactive components such as buttons, control levers, or touch screens do not meet the user's expectations, it is not conducive to the completion of take-over. This is because when the system issues a take-over request, the driver who is performing nondriving tasks needs to quickly switch their attention to the driving situation $[11,12]$. In addition, take-over tasks require the driver to respond in the shortest period. It was found that the lead time affected the take-over quality [13-15]. The interactive components that meet the user's preferences in dimension and space can enable the user to complete the interactions quickly when taking over, which is beneficial to the improvement of take-over performance.

Merat et al. divided driving take-over into two parts: operation and cognition, and found that the driver could recover the operational ability within $1-2 \mathrm{~s}$, but $6-10 \mathrm{~s}$ or even more than $10 \mathrm{~s}$ were needed to recover the cognitive ability [16]. This is related to the driver's response and requires the design of buttons, levers, and other interactive components should conform to the driver's operating characteristics and control force level. When the control right is switched, the driver's response refers to the ability to resume looking at the front in time and operating the vehicle after receiving the take-over request from the system. The response is mainly quantified by a variety of reaction time and take-over time (from the time when the system sends out take-over request to the time when the driver operates the steering wheel or pedals to achieve manual driving) [17]. Gold et al. quantified the reaction time of the driver when taking over and the results showed that the average reaction time of fixation, putting the hand back to the steering wheel, and looking around the rearview mirror was relatively $0.5 \mathrm{~s}$, $1.5 \mathrm{~s}$, and $3 \mathrm{~s}$ [18], which provided a reference for the dimension design of the interactive components.

Making the design of the HMI accord with the user's perceptual judgment is of great significance to a sustainable interaction of human-computer codriving [19]. The dimension and spatial layout of an interface are related to the preference of passengers [20]. The size of the elements on the HMI determines the range of body motion and the user comfort during the take-over process, which is also closely related to usability. Usability is the suitability and efficiency of user-interface interaction [21]. In order to expand the usability of the HMI in the car, the hierarchical interface properties such as the size of the touch keys were studied $[22,23]$. In addition, in terms of take-over performance, the average take-over reaction time of older drivers was at least $1.2 \mathrm{~s}$ longer than that of younger drivers [24], while the driving speed after taking over was lower than that of young drivers [25]. Therefore, the efficiency problem caused by the dimension of the take-over interaction interface is very important, especially now that the aging phenomenon is more and more serious. Research showed that drivers from different age groups had significant differences in perceived safety and intention to use of automated driving and the score increased with age [26].

\subsection{Application of GA in Industrial Design and Ergonomics.} In industrial product design, GA is often used in product gene research. Based on the overall form of the product, the shape is divided into several small feature units until it cannot be divided [27]. At present, related research studies of product gene mostly adopt genetic operations such as crossover and mutation to gradually cultivate a mature scheme after many iterations. Chen et al. obtained the gene expression of the products based on the reverse deduction of functional requirements [28]. In the research of consumers' perception and response to product appearance, GA could be used to find the approximate optimal combination and appraise product appearance and color design [29] and evaluate people's emotion in the design process [30].

GA can solve the noise problem brought about by subjective factors such as product users' cognition and preference. Take the noise source of interactive genetic algorithms (IGA) as an example, Sun analyzed the relationship between cognitive noise and evaluation accuracy [31]. IGA is a branch of evolutionary computing (EC), which can evolve and generate design elements. Kamalian et al. put forward the idea of interval evaluation: users' interaction behaviors interfered with the evolution process in the form of Pareto rank shifting so that one evaluation was enough in the iteration [32]. Gu et al. obtained the user's perceptual evaluation by the neural network so as to achieve faster convergence without increasing the user's burden [33]. 
In terms of the shape and size of HMI, GA could determine the best material characteristics of the product handle interface so as to achieve the optimal mechanical response of the system by the fingertip model of the product handle [34]. In other fields of ergonomics, GA was also a popular method. In order to improve the efficiency and accuracy of human shape prediction, Cheng et al. designed a new prediction method by using GA and BP neural network [35]. The results showed that the prediction effect of this GABP model was better than that of BP, SVM, and $K$-means models, and it could accurately predict and cluster human body shapes. In addition, some research studies had applied a hybrid model based on GA, classification tree, and multivariate adaptive expression splines to accurately predict the most influential risk factors for the upper limb musculoskeletal diseases [36]. Besides, Sana et al. proposed a mathematical model of job scheduling problem for the ergonomic factors of repetitive works, lifting works, and awkward postures in highly variable manufacturing environments [37]. This was a multiobjective optimization problem with comprehensive ergonomic constraints, which was solved by an improved nondominated sorting GA. The results improved the working conditions of $69 \%$ of the workers. GA could also be used to solve the optimization design problem of walking worker manual assembly line [38]. The goal of this study was to generate the required models at the lowest cost while maintaining an ergonomically balanced operation. These studies all provided a basis for applying GA to the evolution of human postures and size.

\section{Methods}

3.1. Equipment and Procedure of the Experiment. In this study, a driving simulator was used as the experiment platform to collect the PCD, task completion time, and error times of the subjects in take-over tasks. In order to modify the controllers according to the optimal dimensions obtained by GA, we chose a nonstandard simple driving simulator (Figure 1). In order to improve the applicability and validity of the data in urban environments, the driving scene of the simulator was set as city roads.

New models of the side stalk and buttons conforming to dimensions with the highest fitness would be made by $3 \mathrm{D}$ printers to replace the original ones. Then, we rewrote the codes to interconnect the printed models with the simulator system. By this means, the task completion time and error times of the subjects under the new HMI could be collected to analyze the differences in the interaction performance between the two HMIs. A 3-spoke steering wheel with a diameter of $\Phi=365 \mathrm{~mm}$ was used on the simulator. We defined the function keys of the main take-over tasks on the steering wheel and the gear lever panel. The subjects were trained to be familiar with the experimental process and the functions of each key.

At present, most mass-produced smart cars in the world were at L0 or L1, while others such as the latest Tesla ModelS/X, Volvo XC90, and Audi A8 were still between L2 and L3 [17]. Therefore, this paper analyzed the interaction modes of the self-adaptive cruise, adjustment of the distance between

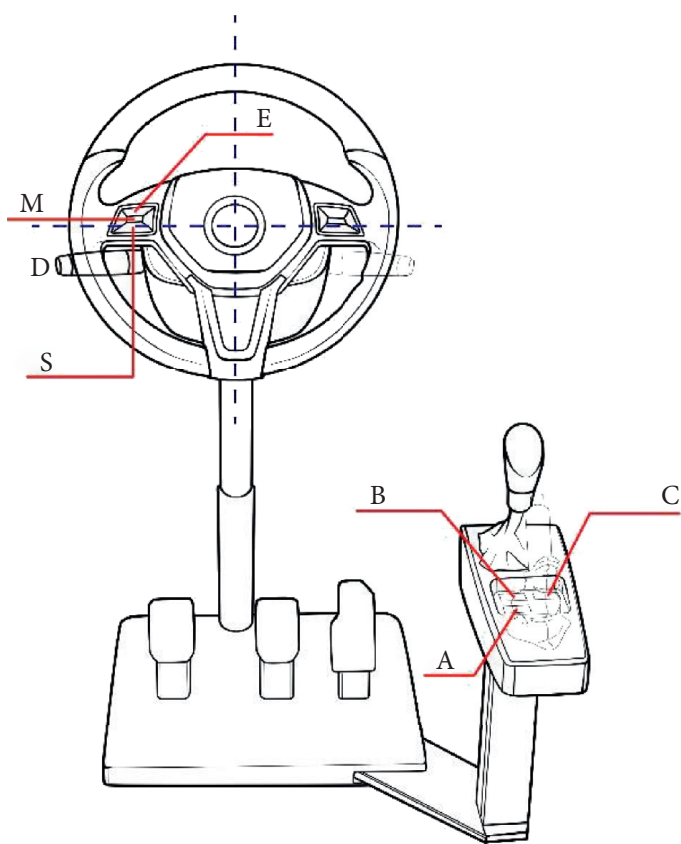

FIGURE 1: Wireframe of the driving simulator and the function keys used in the experiment.

cars, and auto hold, which were core driving assistance functions (DAF) of automated driving between L1-L2. And the PCD reported by the subjects in the process of interaction was collected.

The length of cruise control rods in different vehicles was divergent from each other. And for the cruise switch that controlled by buttons, the distance between the button and the center of the driver or the steering wheel was also different. Due to the large diversity among the dimensions of steering wheels and interior space in multifarious vehicles, it was not significantly meaningful to analyze the absolute sizes of these controllers. Therefore, PCD was collected in this study rather than absolute sizes. That is to take the horizontal and vertical centerlines of the steering wheel as references and collect the distance between the reference line and the perceived comfortable point on the controller. In this way, the dimensions would be according to the user's perceptual judgment and preference. The calibration points and ranges of the 14 dimensions involved in the three DAFs are shown in Table 1.

The automatic transmission was the default option in the experiment, and speed was a controlled variable. Thus, the subjects need not change gear. They were asked to complete the driving process of the whole virtual road section according to the prompts on the laptop screen which was playing interactive animation. The prompts included the task to be performed, the number of times to perform a certain task, and the components to be used in the next task. The task flow of the experiment is shown in Figure 2:

The specific procedures were as follows:

(1) The subjects started the experiment in manual driving mode. When the system first prompted "auto hold," the subjects needed to press the A button on the gear lever panel. 
TABLE 1: The driving assistance functions, interaction modes, and the analyzed dimensions.

\begin{tabular}{lll}
\hline $\begin{array}{l}\text { Driving assistance } \\
\text { functions }\end{array}$ & Interaction modes of take-over tasks & $\begin{array}{l}\text { Dimensions between the interactive component and the } \\
\text { steering wheel center }\end{array}$ \\
\hline
\end{tabular}

(A1) Switch the control right by the side stalk D
(A) Self-adaptive cruise

(A2) Switch the control right by the button $\mathrm{E}$ (a11) The PCD between the left endpoint of the stalk D and the vertical centerline of the steering wheel (VCSW) (a12) The PCD between the left endpoint of the stalk D and the horizontal centerline of the steering wheel (HCSW)

(a21) The PCD between the vertical centerline (VC) of the button E and the VCSW

(a22) The PCD between the horizontal centerline (HC) of the button E and the HCSW

(b11) The PCD between the VC of the button M and the

(B1) Adjust the distance by the button M which was VCSW

in the middle of the left spoke of the steering wheel (b12) The PCD between the HC of the button M and the HCSW

(b21) The PCD between the VC of the button S and the VCSW

(B2) Adjust the distance by the button $S$ which was on the bottom edge of the left spoke of the steering wheel

(C1) Start the function by button A which was under the electric parking brake (EPB) button

(C2) Start the function by button B which was on the VCSW

(b22) The PCD between the HC of the button S and the HCSW

(c11) The PCD between the VC of button A and the

VCSW

(c12) The PCD between the HC of button A and the HCSW

(c21) The PCD between the VC of button B and the

(C) Auto hold left of the EPB button

(c22) The PCD between the HC of button B and the HCSW

(c31) The PCD between the VC of button $\mathrm{C}$ and the

(C3) Start the function by button $\mathrm{C}$ which was on the right of the EPB button
VCSW

(c32) The PCD between the $\mathrm{HC}$ of button $\mathrm{C}$ and the HCSW

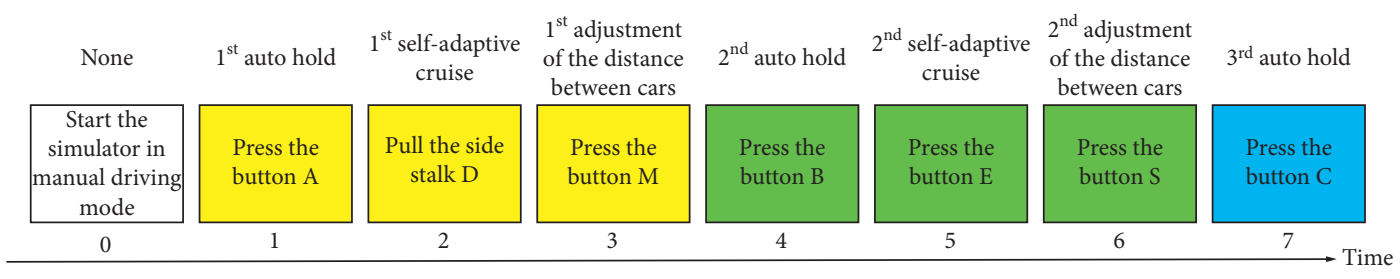

FIGURE 2: The task flow of the experiments.

(2) After stopping stably, the subjects ought to start manual driving again.

(3) When the system first prompted that the selfadaptive cruise function could be used, the subjects turned the cruise control stalk D. This was to simulate switching the manual driving mode to the automated driving mode and entering the state of self-driving. At this moment, the virtual vehicle in the system would run independently according to the preset navigation route.

(4) When the system prompted for adjusting the distance between cars for the first time, the subjects needed to press the $\mathrm{M}$ button.

(5) Then the subjects should complete auto hold, selfadaptive cruise, and distance adjustment in sequence for the second time and pressed the buttons B, E, and $\mathrm{S}$, respectively.

(6) Complete the auto hold operation for the third time and press the $\mathrm{C}$ button.

(7) After the completion of all the above tasks, the subjects were asked to point out the comfortable position of the side stalk and buttons according to the operating experience. A researcher recorded the point with a marker pen on the simulator and calibrated the sizes.

The experiment used a within-subjects design. There were 13 subjects. Each experiment lasted about 3 minutes, and the subjects needed to carry out the above tasks five times to complete the experiment of one day. In order to ensure the accuracy and stability 
of the perceptual experience, the experiment would be in progress for six days. As a result, each subject would carry out the tasks for $5 * 6=30$ times. The PCD data collected each time were taken as the initial population and the dimensions with the highest fitness would be obtained by GA.

(8) In the 30th experiment, the completion time and error times of the subjects during the seven take-over tasks were collected.

(9) After obtaining the PCD data with the highest adaptability, models of the side stalk and buttons were made by $3 \mathrm{D}$ printers to replace the original ones. The subjects were asked to repeat the seven tasks in Figure 2 again with the modified simulator. And their completion time and error times under the new HMI would be collected.

3.2. Information Entropy and Genetic Algorithm. GA is a self-adaptive global optimization algorithm which imitates the natural selection and individual heredity of the biological world [39]. Traditionally, interactive genetic algorithm (IGA) was widely used in product modelling design for subject recognition [40]. However, IGA has some shortcomings, including the uncertainty of individual fitness values, the nonpersistence of the individual evaluation process, and the nonuniqueness of optimization results [41]. In this study, GA was taken as the main research method while PSC was used in the construction of fitness function, which integrated user preference, intuition, emotion, and psychological optimization into it. Therefore, the fitness values of evolutionary individuals could make the population evolve in the direction that users expected.

3.2.1. Fitness Function. Whether the population can evolve in the direction of the ideal solution depends on the establishment of fitness function to a great extent. According to the user's PSC and the weight of the three DAFs that needed the driver to take over, the artificial fitness function was formulated as follows:

$$
F=\frac{1}{\left(\beta_{1} Q_{1}+\beta_{2} Q_{2}+\beta_{3} Q_{3}\right)} .
$$

Among which $\beta_{1} \sim \beta_{3}$ represented the importance weights of the three DAFs, which were obtained by calculating the information entropy. $Q_{1} \sim Q_{3}$ meant the comfortable dimension of the operating components of each DAF listed in Table 1. $Q_{1}=a_{11} * a_{12}+a_{21} * a_{22}, Q_{2}=b_{11} *$ $b_{12}+b_{21} * b_{22}, \quad Q_{3}=c_{11} * c_{12}+c_{21} * c_{22}+c_{31} * c_{32}$. The meanings of $a_{11} \sim c_{32}$ values are shown in Table 1, which were obtained in step (7) of the experiment. For each interaction mode, the comfortable position of the corresponding operation component was identified with a point at first, and then the distances between the point and the horizontal and vertical centerlines of the steering wheel were measured. The two distances were multiplied to get a relative comfort area. $F$ was used to evaluate the fitness degree of individuals. With a smaller size, the user's behavioral path to complete the operations could be shorter, and the task completion time would be relatively less, which were conducive to the completion of the take-over tasks. Therefore, the larger the $F$ value was, the more reasonable the sizes were.

\subsubsection{Information Entropy and the Weight of the Driving} Assistance Functions. Based on the entropy theory, the study explored the importance level of the main DAFs in L1-L2 automated driving and analyzed the influencing factors of users' purchase intentions. The uncertainty degree of the divided sample set, which was measured by means of calculating the information gain, was used as the standard to weigh the quality of the division. The larger the information gain, the less the uncertainty degree of the sample set.

Entropy refers to the degree of system chaos, which is a measurement of the possibility of the system in a certain macroscopic state. Claude Elwood Shannon put forward the concept of information entropy to express the order degree of a system [42]. Let $S$ be a set of $s$ samples. Suppose the classification attribute has $m$ different values: $C_{i}(i=$ $1,2, \ldots, m)$, and let $s_{i}$ be the number of samples in class $C_{i}$. Then, for a given sample, its total entropy is $I\left(s_{1}, s_{2}, \ldots, s_{m}\right)=-\sum_{i=1}^{m} P_{i} \log _{2}\left(P_{i}\right)$. Among which $P_{i}$ is the probability that any sample belongs to $C_{i}$.

Let an attribute $A$ have $k$ different values: $\left\{a_{1}, a_{2}, \ldots, a_{k}\right\}$. Using the attribute to divide the set $S$ into $k$ subsets $\left\{S_{1}, S_{2}, \ldots, S_{k}\right\}$. Among which $S_{j}$ contains the samples with a value $a_{j}$ in the set $S$. Let $s_{i j}$ be the number of samples of class $C_{j}$ in subset $S_{j}$. Then, the information entropy of the samples divided according to $A$ is given by

$$
E(A)=\sum_{j=1}^{k} \frac{s_{1 j}+s_{2 j}+\cdots+s_{m j}}{s} I\left(s_{1 j}, s_{2 j}, \ldots, s_{m j}\right),
$$

where $I\left(s_{1 j}, s_{2 j}, \ldots, s_{m j}\right)=-\sum_{i=1}^{m} P_{i j} \log _{2}\left(P_{i j}\right)$. And $P_{i j}=$ $\left(\left(s_{1 j}+s_{2 j}+\cdots+s_{m j}\right) / s\right)$ is the probability of the samples of class $C_{j}$ in subset $S_{j}$.

Finally, the information gain obtained by dividing the set $S$ according to the attribute $A$ is Gain $(A)=$ $I\left(s_{1}, s_{2}, \ldots, s_{m}\right)-E(A)$. Obviously, the smaller the $E(A)$, the larger the Gain $(A)$, which means more information is provided by $A$ to judge the users' purchase intention and the importance of $A$ is higher. After normalizing the Gain $(A)$ values of the three DAFs, the weight values $\beta_{1} \sim \beta_{3}$ in the fitness function could be formed.

We sent questionnaires online to eight professional users to investigate their purchase decisions of products formed by different combinations of interaction modes. The purchase decisions were divided into two categories which were "buy" and "not buy." Delphi method was used to collect their opinions in an all-round way and ensure the consistency of their decisions. Compared with taking the most frequently selected option as the final decision, the results of the Delphi method were more scientific, and more information was available in the research [43]. 
3.2.3. Genetic Operation and Evolutionary Process. In this system, the premise to debug the genetic operation and make judgment was due to the irrationality and uncertainty of the shape and spatial dimension design of automobile controllers and panels, the dimension evolution driven by users' expectation was not only to search for the optimal solution but also to obtain the satisfactory solution under limited resources. Therefore, the model was different in coding, selection, crossover, and mutation from traditional GA, which was to solve problems represented by explicit functions.

(1) Coding. Generally, there are two kinds of coding methods for optimization problems which are real number coding and binary coding. And both have advantages and disadvantages. Mapping errors exist in traditional binary coding when continuous functions are discretized. When the individual coding string is short, the accuracy requirements may not be met. While when the string is long, although the coding accuracy can be improved, the search space of GA will expand dramatically [44]. Therefore, we used real number coding in this study.

In the process of evolution, a population $\operatorname{Pop}(t)$ was formed. Each chromosome $X_{i}^{t}$ in the population represented data from one of the 30 experiments. The genes in the chromosome were composed of PCD reported by the subjects after completing the whole experiment process, which could be expressed as follows:

$$
\begin{aligned}
\operatorname{Pop}(t) & =\left\{X_{1}^{t}, X_{2}^{t}, \ldots X_{i}^{t}, \ldots, X_{M}^{t}\right\} \\
X_{i}^{t} & =\left[\theta_{i 1}^{t}, \theta_{i 2}^{t}, \ldots, \theta_{i b}^{t}, \ldots, \theta_{i N}^{t}\right]
\end{aligned}
$$

where $t$ represented the generation number of evolutions, $\theta$ represented gene coding, and $M$ represented the population size.

(2) Initialized Population and Selection. The population needed to be initialized before running GA. According to the research demand, PCD data from the 30 experiments were taken as the primary population. Each time, data of the 14 PCD indexes were collected to form the population matrix.

The selection operation was the process of selecting individuals from the previous generation of the population to form the next generation. The purpose of selection was to obtain fine individuals based on the fitness values so that they would have the opportunity to reproduce as parents for the next generation. Individuals with high fitness were more likely to be inherited, while the ones with low fitness were less likely. After debugging and comparison, we chose the method of normalized geometric select (NGS) to perform the selection operation. NGS was mainly to sort the fitness values, and the better individuals would be maintained as parents. This was more suitable for the selection of product design schemes and also helpful to prevent better individuals from being damaged.

(3) Crossover and Mutation. By crossover operation, a new generation of individuals could be obtained, which combined the characteristics of their parents and embodied the idea of information exchange. In the case of real number coding, the arithmetic uniform crossover (AUC) between individuals was generally utilized [45]. AUC was a linear combination of two individuals to produce two new ones. The method of crossover and getting the next generation was as follows:

$$
\begin{aligned}
& c 1=p 1 * a+p 2 *(1-a), \\
& c 2=p 1 *(1-a)+p 2 * a,
\end{aligned}
$$

where $p 1$ and $p 2$ were the parents and $a$ was a random mix amount.

In this study, the nonuniform mutation (NM) was used to perform the mutation operation. Let an individual be $X=X_{1} X_{2}, \ldots, X_{k}, \ldots, X_{l}$. If $X_{k}$ was the variation point and its value range was $\left[U_{\min }^{k}, U_{\max }^{k}\right]$, a new individual $X=X_{1} X_{2}, \ldots, X_{k}^{\prime}, \ldots, X_{l}$ could be obtained after nonuniformly mutating at this point. And the new gene value was given by

$$
X_{k}^{\prime}= \begin{cases}X_{k}+\Delta\left(t, U_{\max }^{k}-X_{k}\right), & \text { if } \operatorname{random}(0,1)=0, \\ X_{k}-\Delta\left(t, X_{k}-U_{\min }^{k}\right), & \text { if } \operatorname{random}(0,1)=1,\end{cases}
$$

where $\Delta(t, y)$ was a random number conforming to the nonuniform distribution in the range $[0, y] . y$ represented $U_{\max }^{k}-X_{k}$ and $X_{k}-U_{\min }^{k}$. It was required that with the increase of evolutionary generation number $t$, the probability of $\Delta(t, y)$ approaching to 0 also raised gradually.

In this study, $\Delta(t, y)$ was defined as follows:

$$
\Delta(t, y)=y\left(1-r^{(1-t / T) b}\right),
$$

where $r$ was a random number conforming to the nonuniform distribution in the range $[0,1]$, which was the selection pressure. $T$ was the maximum number of evolutionary generations, in this study $T=25$. In the 25 iterations, the better individuals with high fitness could be found out to explore the evolutionary mechanism of the appropriate HMI dimensions of the DAFs. $b$ was a parameter to adjust the variable step size, which was a system parameter. It determined the dependence degree of the random number disturbance on $t$, and the range was usually 2 5. After debugging the model and making comparisons repeatedly, this parameter was set as $b=4$ in this study.

\section{Results}

\subsection{Information Entropy and the Weights of the DAFs}

4.1.1. The Expectation Information for the Classification of the HMI of Take-Over Tasks. According to different combinations of the seven interaction modes in Table 1 , a total of $2 * 2 * 3=12$ HMI schemes could be generated. By means of Delphi method, purchase decisions for the 12 schemes were investigated. After four rounds of expert investigations, the purchase decisions of the eight professional users reached an agreement. The classification results are shown in Table 2.

According to the purchase intentions of the professional users, 12 HMI schemes could be divided into two categories: 
TABLe 2: Classification of purchase intentions to interaction modes.

\begin{tabular}{|c|c|c|c|}
\hline $\begin{array}{l}\text { The starting mode of self- } \\
\text { adaptive cruise }\end{array}$ & $\begin{array}{l}\text { The position of the function key to adjust the } \\
\text { distance between cars }\end{array}$ & $\begin{array}{l}\text { The position of the function key of } \\
\text { auto-hold }\end{array}$ & $\begin{array}{l}\text { Purchase } \\
\text { decision }\end{array}$ \\
\hline Pulling the side stalk & On the middle of the left spoke & Under the EPB button & 2 \\
\hline Pressing the button & On the middle of the left spoke & Under the EPB button & 2 \\
\hline Pulling the side stalk & On the bottom edge of the left spoke & Under the EPB button & 2 \\
\hline Pressing the button & On the bottom edge of the left spoke & Under the EPB button & 1 \\
\hline Pulling the side stalk & On the middle of the left spoke & On the left of the EPB button & 2 \\
\hline Pressing the button & On the middle of the left spoke & On the left of the EPB button & 1 \\
\hline Pulling the side stalk & On the bottom edge of the left spoke & On the left of the EPB button & 2 \\
\hline Pressing the button & On the bottom edge of the left spoke & On the left of the EPB button & 2 \\
\hline Pulling the side stalk & On the middle of the left spoke & On the right of the EPB button & 1 \\
\hline Pressing the button & On the middle of the left spoke & On the right of the ЕРB button & 1 \\
\hline Pulling the side stalk & On the bottom edge of the left spoke & On the right of the EPB button & 2 \\
\hline Pressing the button & On the bottom edge of the left spoke & On the right of the EPB button & 1 \\
\hline
\end{tabular}

$U_{1}=1$ (buy) and $U_{2}=2$ (not buy). By summarizing and analyzing the data, the probabilities of the two categories were as follows: $P\left(U_{1}\right)=(5 / 12)$ and $P\left(U_{2}\right)=(7 / 12)$. Based on the formula, the total entropy was as follows:

$$
I(U)=-\frac{5}{12} \log _{2} \frac{5}{12}-\frac{7}{12} \log _{2} \frac{7}{12}=0.9799 .
$$

\subsubsection{The Conditional Entropy and Information Gain of Each} Influencing Factor. In this study, there were three factors that determined the purchase intentions of users. $A_{1}$ : the starting mode of the self-adaptive cruise, $A_{2}$ : the position of the function key to adjust the distance between cars, and $A_{3}$ : the position of the function key of auto hold. The probabilities of the factors and each condition are shown in Table 3 .

The information entropy values of the three factors were as follows:

$$
\begin{aligned}
& E\left(A_{1}\right)=\frac{6}{12} I(1,5)+\frac{6}{12} I(4,2)=0.7842, \\
& E\left(A_{2}\right)=\frac{6}{12} I(3,3)+\frac{6}{12} I(2,4)=0.9592 \\
& E\left(A_{3}\right)=\frac{4}{12} I(1,3)+\frac{4}{12} I(1,3)+\frac{4}{12} I(3,1)=0.8113 .
\end{aligned}
$$
follows:

Therefore, the information gains of the factors were as

$$
\begin{aligned}
& \text { Gain }\left(A_{1}\right)=I(U)-E\left(A_{1}\right)=0.1957, \\
& \text { Gain }\left(A_{2}\right)=I(U)-E\left(A_{2}\right)=0.0207, \\
& \text { Gain }\left(A_{3}\right)=I(U)-E\left(A_{3}\right)=0.1686 .
\end{aligned}
$$

According to the information gains, the starting mode of the self-adaptive cruise was the most important for the user's perceived comfort and purchase intension, while the position of the function key to adjust the distance between cars was not so significant. By normalizing the information gains of each influencing factor, the results were taken as the weights of the factors, which were
TABle 3: The probabilities of the three factors and each condition.

\begin{tabular}{lccc}
\hline & $P\left(V_{i}\right)$ & $P\left(U_{1} \mid V_{i}\right)$ & $P\left(U_{2} \mid V_{i}\right)$ \\
\hline$A_{1}$ & & & \\
Side stalk & $6 / 12$ & $1 / 6$ & $5 / 6$ \\
Button & $6 / 12$ & $4 / 6$ & $2 / 6$ \\
$A_{2}$ & & & \\
Middle & $6 / 12$ & $3 / 6$ & $3 / 6$ \\
Bottom edge & $6 / 12$ & $2 / 6$ & $4 / 6$ \\
$A_{3}$ & & \\
Under the EPB button & $4 / 12$ & $1 / 4$ & $3 / 4$ \\
On the left of the EPB button & $4 / 12$ & $1 / 4$ & $3 / 4$ \\
On the right of the EPB button & $4 / 12$ & $3 / 4$ & $1 / 4$ \\
\hline
\end{tabular}

$P\left(V_{i}\right)$ represents the probability of each condition of the influencing factors. $P\left(U_{1} \mid V_{i}\right)$ and $P\left(U_{2} \mid V_{i}\right)$, respectively, indicate the conditional probabilities of the two categories.

$$
\begin{aligned}
& \beta_{1}=\frac{\text { Gain }\left(A_{1}\right)}{\text { Gain }\left(A_{1}\right)+\text { Gain }\left(A_{2}\right)+\text { Gain }\left(A_{3}\right)}=0.5083, \\
& \beta_{2}=\frac{\text { Gain }\left(A_{2}\right)}{\text { Gain }\left(A_{1}\right)+\text { Gain }\left(A_{2}\right)+\text { Gain }\left(A_{3}\right)}=0.0538, \\
& \beta_{3}=\frac{\text { Gain }\left(A_{3}\right)}{\text { Gain }\left(A_{1}\right)+\text { Gain }\left(A_{2}\right)+\text { Gain }\left(A_{3}\right)}=0.4379 .
\end{aligned}
$$

After taking these values into formula (1), the artificial fitness function could be constructed as follows:

$$
F=\frac{1}{\left(0.5083 * Q_{1}+0.0538 * Q_{2}+0.4379 * Q_{3}\right)} \text {. }
$$

\subsection{Results of $G A$}

4.2.1. Characteristics of the Population. The population size of this study was Pop $=30$. For the convenience of recording, the unit of each dimension was decimeter $(\mathrm{dm})$. The features of the 14 dimensions are described in Table 4 .

4.2.2. Solving Process of GA. To match the user's expectation, the seed of each initial population should be able to 
TABle 4: Features of the 14 dimensions.

\begin{tabular}{lccccc}
\hline $\begin{array}{l}\text { Tasks and } \\
\text { dimensions }\end{array}$ & $\begin{array}{c}\text { Mean } \\
(\mathrm{dm})\end{array}$ & $\begin{array}{c}\text { Std. } \\
\text { deviation }\end{array}$ & $\begin{array}{c}\text { Minimum } \\
(\mathrm{dm})\end{array}$ & $\begin{array}{c}\text { Maximum } \\
(\mathrm{dm})\end{array}$ \\
\hline \multirow{2}{*}{$\mathrm{A} 1$} & $\mathrm{a} 11$ & 1.9513 & 0.0789 & 1.8086 & 2.091 \\
& $\mathrm{a} 12$ & 0.6055 & 0.063 & 0.5117 & 0.6977 \\
\hline \multirow{2}{*}{$\mathrm{A} 2$} & $\mathrm{a} 21$ & 0.8624 & 0.0242 & 0.8224 & 0.8979 \\
& $\mathrm{a} 22$ & 0.0784 & 0.0477 & 0.0008 & 0.1487 \\
\hline \multirow{2}{*}{$\mathrm{B} 1$} & $\mathrm{~b} 11$ & 1.3356 & 0.0633 & 1.2528 & 1.4475 \\
& $\mathrm{~b} 12$ & 0.0315 & 0.0169 & 0.0024 & 0.0588 \\
\hline \multirow{2}{*}{$\mathrm{B} 2$} & $\mathrm{~b} 21$ & 1.3999 & 0.0526 & 1.3060 & 1.4952 \\
& $\mathrm{~b} 22$ & 0.322 & 0.0139 & 0.3011 & 0.3497 \\
\hline \multirow{2}{*}{$\mathrm{C} 1$} & $\mathrm{c} 11$ & 2.9776 & 0.1254 & 2.8032 & 3.1940 \\
& $\mathrm{c} 12$ & 3.272 & 0.1414 & 3.0207 & 3.4928 \\
\multirow{2}{*}{$\mathrm{C} 2$} & $\mathrm{c} 21$ & 2.7451 & 0.0305 & 2.7010 & 2.7966 \\
& $\mathrm{c} 22$ & 3.2714 & 0.1304 & 3.0143 & 3.4819 \\
\multirow{2}{*}{$\mathrm{C} 3$} & $\mathrm{c} 31$ & 3.2929 & 0.0596 & 3.2019 & 3.3963 \\
& $\mathrm{c} 32$ & 3.244 & 0.1523 & 3.0011 & 3.4932 \\
\hline
\end{tabular}

cover part of the information of the PCD data. And it should be capable of satisfying the geometric description rules of product evolution in terms of space and size [46]. The initial population of this paper came from repeated experiments in many days, and the perceptual experience and spatial attributes reflected by the data were stable. The optimal solution of the model was obtained after 25 iterations. Figure 3 shows the evolutionary process of GA.

In the figure, the red line represents the best fitness value of each generation. And the blue dotted line describes the mean fitness of each generation. It could be seen from the figure that the optimal value in the model increased with the number of iterations and converged into a straight line after reaching the maximum value. Until the optimal value of the model remained unchanged, the global optimal solution could be obtained.

The genetic evolution stopped after 25 iterations. It could be seen from the figure that although the deviation between the optimal fitness values and the mean ones increased after the 9th iteration, it continued to evolve, which met the expectation of evolution. After the 21st iteration, the deviation was close to 0 .

In the 25 generations of evolutionary iteration based on the PCD data, eight individuals with the highest fitness were obtained, which were the HMI dimension schemes that met the users' expectation best in a certain generation of the population. It would be rational to make designs of HMIs such as steering wheels and gear lever panels in the later stage according to these fine values. This could not only inherit the modelling genes of the HMIs but was also coupled to the user expectation to a certain extent. The finally generated dimensional data of the individuals with the highest fitness are shown in Table 5.

4.3. Significance of the Difference between the Original Dimensions and the Evolved Ones. To further validate whether the individuals with the highest fitness could bring about a

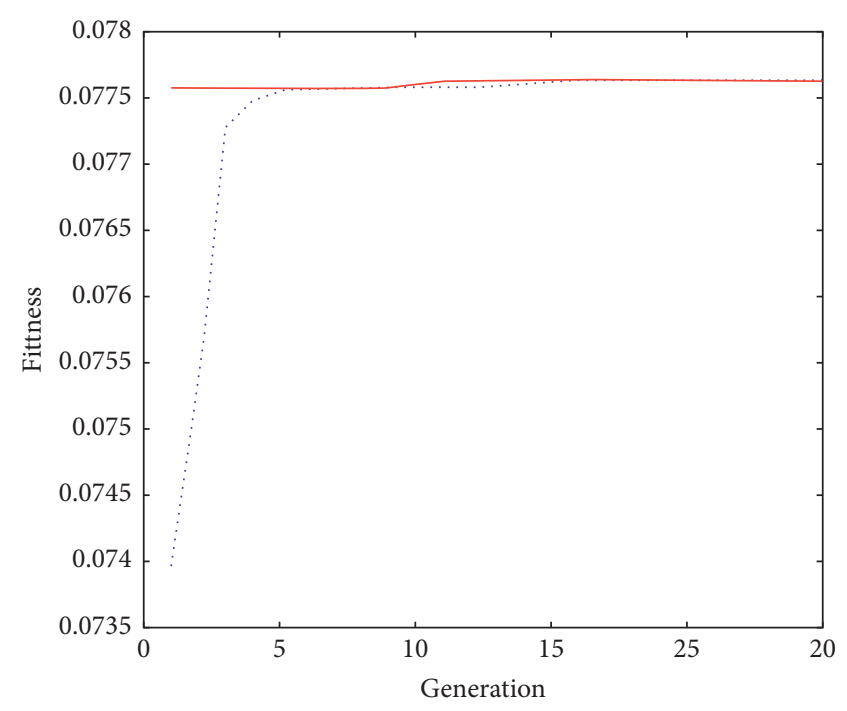

..... Mean fitness

Best fitness

Figure 3: Solving process of GA.

better take-over performance, it was reasonable to collect the needed time to complete driving assistant tasks under the HMI with the original sizes and the one with the evolved sizes. And the differences between the two sets of HMI dimensions could be analyzed.

In the last of the 30 experiments, we collected the time of the 13 subjects to complete the take-over tasks under the seven interaction modes in Table 1. Since the tasks had been repeated many times, the subjects were skilled, and the task completion time would be relatively stable. After making 3D printing models of the side stalk and the buttons according to the most suitable dimensions (Table 5), the driving simulator was reformed. Then the 13 subjects were invited to repeat the seven tasks with the new HMI, and this set of completion time was collected. To judge whether the dimensions could effectively improve the interaction performance and efficiency of driving information processing, paired-sample $t$-test was used on the two groups of data. The results are shown in Table 6.

It could be seen from the results that except for $\mathrm{A} 2$ and $\mathrm{C} 2$, the completion time under the two sets of dimensions in the process of the other five tasks all had a significant difference (Figure 4).

Figure 4 shows that the completion time of each task was shorter when the dimensions with high fitness were used on the HMI. This indicated that the driver's information processing was faster, which helped to improve the quality of take-over and the ability to control the car. As the driver's attention to the HMI might compete with the execution of driving tasks for cognitive resources [47] and impair the driving performance, a shorter completion time of take-over tasks was conducive to the distribution of the driver's psychological resources and more beneficial to traffic efficiency.

It could be found by comparing the completion time of each task under the new HMI that (A) for starting the self- 
TABLE 5: The finally generated dimensional data of the individuals with the highest fitness.

\begin{tabular}{lllllllllllllll}
\hline $\begin{array}{l}\text { (A) The starting mode of self- } \\
\text { adaptive cruise }\end{array}$ & \multicolumn{4}{c}{$\begin{array}{c}\text { (B) The position of the } \\
\text { function key to adjust the } \\
\text { distance between cars }\end{array}$} & \multicolumn{1}{c}{ (C) The position of the function key of auto- } \\
hold
\end{tabular}

TABLE 6: The paired sample $t$-test results of task completion time under the two HMIs with different dimensions.

\begin{tabular}{|c|c|c|c|c|c|c|}
\hline \multirow[t]{2}{*}{ Interaction modes of take-over tasks } & \multicolumn{2}{|c|}{$\begin{array}{l}\text { The task completion } \\
\text { time under the original } \\
\text { dimensions (s) }\end{array}$} & \multicolumn{2}{|c|}{$\begin{array}{l}\text { The task completion } \\
\text { time under the evolved } \\
\text { dimensions (s) }\end{array}$} & \multirow[t]{2}{*}{$t$-value } & \multirow[t]{2}{*}{ Sig. } \\
\hline & Mean & Std. dev. & Mean & Std. dev. & & \\
\hline $\mathrm{A} 1 *$ & 0.715 & 0.185 & 0.537 & 0.167 & 2.553 & 0.025 \\
\hline A2 & 0.657 & 0.181 & 0.637 & 0.186 & 1.151 & 0.272 \\
\hline $\mathrm{B} 1 *$ & 0.648 & 0.13 & 0.462 & 0.206 & 2.797 & 0.016 \\
\hline $\mathrm{B} 2 * *$ & 0.736 & 0.106 & 0.571 & 0.124 & 3.116 & 0.009 \\
\hline $\mathrm{C} 1 *$ & 0.753 & 0.295 & 0.562 & 0.222 & 2.515 & 0.027 \\
\hline $\mathrm{C} 2$ & 0.667 & 0.226 & 0.522 & 0.176 & 1.999 & 0.069 \\
\hline $\mathrm{C} 3 *$ & 0.69 & 0.208 & 0.537 & 0.225 & 2.355 & 0.036 \\
\hline
\end{tabular}

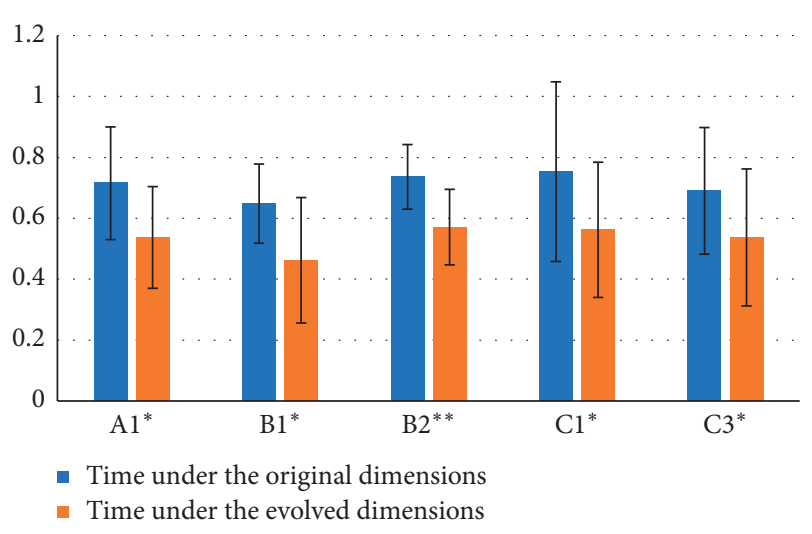

FIGURE 4: The completion time of the tasks with significant differences under the two HMIs with different dimensions.

adaptive cruise, the completion time of operating with a side stalk was shorter; (B) for the task of adjusting the distance between cars, the completion time would be shorter when operating with a button located in the middle of the steering wheel spoke; (C) for the task of auto hold, a starting button on the left side of the EPB button could bring about a shorter completion time. These results provided a reference for the future automobile interior design.

\section{Discussion}

Driving assistant system (DAS) plays an important role in the context of human-computer codriving. As reported, DAS can help the driver to complete the intense driving tasks effectively and abate the pressure level of the driver [48]. Therefore, it is meaningful to make a study for the controller dimensions in the DAS. The study collected comfortable dimensions perceived by the drivers in a relaxed state. The aim was to detect a set of reasonable dimensions which could make drivers at a low level of stress. In addition, it is valuable for drivers to adapt to the different road environment and realize sustainable traffic to optimize the sizes of interactive components on the in-vehicle HMI. The main goals of in-vehicle technologies and cooperative services were to reduce traffic congestion and improve road safety [4].

The study attempted to analyze the optimal HMI dimensions suitable for take-over tasks. The involved HMI components such as the steering wheel, self-adaptive cruise lever, distance adjustment button, and auto hold button were all direct design elements for users to see and touch. Because the design and performance of a product were important factors affecting the decision-making of consumers [49], we calculated the information entropy and information gains of the three kinds of take-over tasks for classifying users' purchase intentions. The information transmitted by a product was multivariant, complex, and fuzzy so that there would be dissipation in the process of transmission. Therefore, the image evaluation process of products could be regarded as a chaotic state. The feedback information could be calculated by information entropy to carry out a comprehensive evaluation. The smaller the entropy of an image, the clearer it was. From the perspective of chaos degree, the 2 nd factor in Table 3 (the position of the function key to adjust the distance between cars) brought more uncertainty to the purchase decision $\left(E\left(A_{2}\right)=0.9592\right)$, which indicated that little information was provided by the 2 nd factor for judging the users' decisions. On the contrary, the uncertainty produced by the 1st factor (the starting mode of the self-adaptive cruise) was the smallest $\left(E\left(A_{1}\right)=0.7842\right)$. This illustrated that using the side stalk or a button to complete the switch of the control right had a great impact on the satisfaction and purchase decision of users.

After normalizing the information entropy of three kinds of take-over tasks, their weight values were calculated, 
and the fitness function was constructed. In this way, the dimensions with the highest fitness could be found out by means of GA.

The background and driving experience of the subjects were different, and their familiarity with the take-over tasks and the HMI was variant. Besides, the noise of users' subjective perception data was always high. Taking the above factors into consideration, we collected PCD data repeatedly from the same 13 subjects to form the initial population. From the evolutionary trajectory, it could be found that in the 8 th, 10th, 11 th, 14 th, $17^{\text {th }}$, and 21st iteration, there appeared an optimal solution.

GA was always used for parameter optimization by searching the optimal solutions while seldom used in user research and environment perception analysis. But in studies of user-centred design, it could search the set of satisfactory solutions driven by user expectations [46]. Many pieces of research had utilized GA to improve the ergonomic efficiency or optimize interactive component sizes in an operating environment [34-38, 50, 51]. In this study, we reported a driver perception research, which investigated the most comfortable HMI sizes under different interaction modes of take-over tasks. By means of normalized geometric selection, arithmetic uniform crossover, and nonuniform mutation, GA was applied in the research of perceived comfortable dimensions and a set of reasonable dimensions was obtained. The dimensional evolutionary method discussed in this study embodied the concept of dynamic interaction design, which took the mutual influence and restrictions among different dimensions into consideration. Compared with traditional methods of measuring many times and taking the average, this method was more advanced. And the study enriched theoretical researches in the field of product design and collaborative optimization.

Perceived comfort was a complex problem for its difficulty to be quantified objectively. But it was useful in ergonomic explorations. Researchers had found that the perceptual messages from product property and usage requirements were competent to the analysis of user experience [52]. Therefore, it was reasonable to probe suitable dimensions by perceived comfort. For the study of HMI design effect in a specific environment, these data could produce important and dependable results [53]. Rational dimensions of the automobile interior HMI played an important role in the driver's operation quality. Caruso [54] studied drivers' experience on the seat by an experiment and found that interior space influenced the drivers' ergonomic performance [54]. For the take-over tasks in the situation of human-computer codriving, the dimensions also affected whether the driver could safely achieve the control right switch in the shortest possible time.

In this paper, paired-sample $t$-test was used to test whether the sizes with the highest fitness could bring about a significant reduction in task completion time. In the results, we found that when the interface adopted the finally evolved sizes, the completion time of each task was shorter. As the completion time of in-vehicle interactive tasks was related to the vehicle speed and driver errors under the context of human-computer codriving [21,55], a shorter completion time would help the driver to control the speed better.

\section{Limitation}

The study explored the reasonable dimensions of the human-machine interface in intelligent cars. Nevertheless, there were still some deficiencies in the equipment used in this study. The driving simulator was easier to control and reform and helped to reduce the subjects' pressure. However, the immersion, visual interactivity, and fidelity degree were not high enough. After obtaining reliable conclusions in this study, outdoor experiment with a real vehicle could be conducted in the next stage.

Besides, the sample size of this study was limited. When the sample size was larger in future studies, the representativeness of the data would be better, and the results of GA could be further improved.

Finally, users' perception of the HMI dimensions deviated from each other. In the next phase of the study, electroencephalography (EEG) signal in the process of performing the driving tasks could be used. By this means, the subjects' attention and intention when interacting with the HMI could be reflected in an objective way $[56,57]$ as a supplement to their subjective report.

\section{Conclusion}

For HMI design of industrial products, physical concrete modelling parameters such as the product shape, size, and layout were the objective properties that users could directly perceive and the basis for the product to be expressed. Based on the dynamic measurement principle, this paper proposed an optimized design method for ergonomic dimensions by GA. The results indicated that the method was effective and provided a new way for product shape innovation and human-machine dimensional automatic design. The main conclusions of this study were as follows:

(1) Based on the information entropy theory, the weight values of the three driving assistance functions were calculated. The results showed that the information gain of the starting mode of the self-adaptive cruise was highest while the information gain of the position of the function key to adjust the distance between cars was lowest, which provided a theoretical basis for the dimensional fitness evaluation mechanism of the HMI.

(2) The evolutionary process of HMI dimensions driven by user expectation was studied, and the evolution conditions were analyzed. The study confirmed the feasibility of normalized geometric selection, arithmetic uniform crossover, and nonuniform mutation in iterative researches for human-machine dimensions. Through parameter debugging, this evolutionary method was proved to be effective in obtaining appropriate sizes of HMI components. The model could make full use of the advantages of GA to improve the user research performance, such as few restrictions on the optimization, high robustness, and an easily achieved global search. 
(3) After finding the dimensions with the highest fitness and applying them to the design of the HMI, withinsubjects experiments showed that the completion time of five take-over tasks was significantly shorter, except for that of Task A2 and C2. This indicated that the information processing of drivers under the HMI with evolved dimensions was faster, which was more beneficial to the vehicle control and take-over quality. Intelligent vehicles achieved intelligent information exchange with people, vehicles, and roads through in-vehicle sensor system and information terminals. Therefore, the evolved dimensions could make the vehicle apt to respond to the environment and have more time to fully deal with the complex traffic environment and would help to improve the traffic efficiency therefrom.

\section{Data Availability}

The data used to support the findings of this study are available from the corresponding author upon request.

\section{Consent}

The study respected and guaranteed the subjects' right to participate in the study and allowed them to withdraw from the study unconditionally at any stage. The study strictly followed the informed consent procedure. The subjects were asked to sign a consent form to inform them of the purpose, process, duration of the experiment, matters needing attention, and the methods and purpose of collecting the data, so as to reduce their anxiety and confusion. In the experiment, the personal safety and health of the subjects took priority. According to the usage guidelines of the driving simulator, we tried to avoid any possible injury in driving tasks. The privacy of the subjects was strictly protected. The subjects were informed of the storage, use, and confidentiality measures of their personal information and image data in advance truthfully. And we would not disclose the information to a third party or spread them on the Internet without authorization.

\section{Conflicts of Interest}

The authors declare that they have no conflicts of interest.

\section{Acknowledgments}

This research was funded by Beijing Urban Governance Research Project (grant no. 20XN244), Scientific Research Program of Beijing Education Commission (grant no. KM202010009003), Yuyou Talent Support Program of North China University of Technology (grant no. 107051360018XN012/018), Tianjin Youth Talents Reserve Support Program, and Chinese Ergonomics Society \& Kingfar Joint Research Fund for Outstanding Young Scholars (grant number CES-Kingfar-2019-001).

\section{References}

[1] S. Jin, J. Yang, E. Wang, and J. Liu, "The influence of highspeed rail on ice-snow tourism in north eastern China," Tourism Management, vol. 78, Article ID 104070, 2020.

[2] J. Yang, R. Yang, J. Sun, T. Huang, and Q. Ge, "The spatial differentiation of the suitability of ice-snow tourist destinations based on a comprehensive evaluation model in China," Sustainability, vol. 9, no. 5, Article ID 774, 2017.

[3] W.-Y. Chung, T.-W. Chong, and B.-G. Lee, "Methods to detect and reduce driver stress: a review," International Journal of Automotive Technology, vol. 20, no. 5, pp. 1051-1063, 2019.

[4] H. Farah and H. N. Koutsopoulos, "Do cooperative systems make drivers' car-following behavior safer?" Transportation Research Part C: Emerging Technologies, vol. 41, pp. 61-72, 2014.

[5] SAE, Taxonomy and Definitions for Terms Related to Driving Automation Systems for On-Road Motor Vehicles SAE J3016TM, Warrendale, PA, USA, 2018.

[6] J. Hoegg, J. W. Alba, and D. W. Dahl, “The good, the bad, and the ugly: influence of aesthetics on product feature judgments," Journal of Consumer Psychology, vol. 20, no. 4, pp. 419-430, 2010.

[7] M. Kyriakidis, R. Happee, and J. C. F. De Winter, "Public opinion on automated driving: results of an international questionnaire among 5000 respondents," Transportation Research Part F: Traffic Psychology and Behaviour, vol. 32, pp. 127-140, 2015.

[8] F. Meng and C. Spence, "Tactile warning signals for in-vehicle systems," Accident Analysis \& Prevention, vol. 75, pp. 333346, 2015.

[9] J. Wan and C. Wu, "The effects of lead time of take-over request and nondriving tasks on taking-over control of automated vehicles," IEEE Transactions on Human Machine Systems, vol. 48, no. 6, pp. 582-591, 2018.

[10] S. Petermeijer, P. Hornberger, I. Ganotis, J. D. Winter, and K. Bengler, "The design of a vibrotactile seat for conveying take-over requests in automated driving," in Proceedings of the 8th International Conference on Applied Human Factors and Ergonomics, Los Angeles, CA, USA, July 2017.

[11] T. Ito, A. Takata, and K. Oosawa, "Time required for take-over from automated to manual driving," in Proceedings of the SAE 2016 World Congress and Exhibition, Detroit, MI, USA, April 2016.

[12] K. Zeeb, A. Buchner, and M. Schrauf, "What determines the take-over time? An integrated model approach of driver takeover after automated driving," Accident Analysis \& Prevention, vol. 78, pp. 212-221, 2015.

[13] B. Mok, M. Johns, K. J. Lee et al., "Emergency, automation off: unstructured transition timing for distracted drivers of automated vehicles," in Proceedings of the IEEE International Conference on Intelligent Transportation Systems, Las Palmas, Spain, September 2015.

[14] S. Samuel, A. Borowsky, S. Zilberstein, and D. L. Fisher, "Minimum time to situation awareness in scenarios involving transfer of control from an automated driving suite," Transportation Research Record Journal of the Transportation Research Board, vol. 2602, no. 2602, pp. 115-120, 2016.

[15] W. Vlakveld, N. van Nes, J. de Bruin, L. Vissers, and M. van der Kroft, "Situation awareness increases when drivers have more time to take over the wheel in a level 3 automated 
car: a simulator study," Transportation Research Part F: Traffic Psychology and Behaviour, vol. 58, pp. 917-929, 2018.

[16] N. Merat, A. H. Jamson, F. C. H. Lai, M. Daly, and O. M. J. Carsten, "Transition to manual: driver behaviour when resuming control from a highly automated vehicle," Transportation Research Part F: Traffic Psychology and Behaviour, vol. 27, no. 26, pp. 274-282, 2014.

[17] C. Z. Wu, H. R. Wu, and N. C. Lyu, "Review of control switch and safety of human-computer driving intelligent vehicle," Journal of Traffic and Transportation Engineering, vol. 18, no. 6, pp. 131-141, 2018.

[18] C. Gold, D. Damböck, L. Lorenz, and K. Bengler, “"'Take over!" how long does it take to get the driver back into the loop?" Proceedings of the Human Factors and Ergonomics Society Annual Meeting, vol. 57, no. 1, pp. 1938-1942, 2013.

[19] H. Yang, Y. Zhao, and Y. Wang, "Identifying modeling forms of instrument panel system in intelligent shared cars: a study for perceptual preference and in-vehicle behaviors," Environmental Science and Pollution Research, vol. 27, no. 1, pp. 1009-1023, 2020.

[20] A. Berkovich, A. Lu, B. Levine, and A. V. Reddy, "Observed customer seating and standing behavior and seat preferences on board subway cars in New York city," Transportation Research Record: Journal of the Transportation Research Board, vol. 2353, no. 1, pp. 33-46, 2013.

[21] R. Li, Y. V. Chen, C. Sha, and Z. Lu, "Effects of interface layout on the usability of in-vehicle information systems and driving safety," Displays, vol. 49, pp. 124-132, 2017.

[22] H. Kim, S. Kwon, J. Heo, H. Lee, and M. K. Chung, "The effect of touch-key size on the usability of in-vehicle information systems and driving safety during simulated driving," Applied Ergonomics, vol. 45, no. 3, pp. 379-388, 2014.

[23] H. Kim and H. Song, "Evaluation of the safety and usability of touch gestures in operating in-vehicle information systems with visual occlusion," Applied Ergonomics, vol. 45, no. 3, pp. 789-798, 2014.

[24] M. Koerber, C. Gold, D. Lechner, and K. Bengler, "The influence of age on the take-over of vehicle control in highly automated driving," Transportation Research Part F: Traffic Psychology \& Behaviour, vol. 39, pp. 19-32, 2016.

[25] H. Clark, A. C. Mclaughlin, B. Williams, and J. Feng, "Performance in takeover and characteristics of non-driving related tasks during highly automated driving in younger and older drivers," Proceedings of the Human Factors \& Ergonomics Society Annual Meeting, vol. 61, no. 1, pp. 37-41, 2017.

[26] I. Lijarcio, S. A. Useche, J. Llamazares, and L. Montoro, "Perceived benefits and constraints in vehicle automation: data to assess the relationship between driver's features and their attitudes towards autonomous vehicles," Data in Brief, vol. 27, Article ID 104662, 2019.

[27] K.-Z. Chen and X.-A. Feng, "Virtual genes of manufacturing products and their reforms for product innovative design," Proceedings of the Institution of Mechanical Engineers, Part C: Journal of Mechanical Engineering Science, vol. 218, no. 5, pp. 557-574, 2004.

[28] K.-Z. Chen, X.-A. Feng, and X.-C. Chen, "Reverse deduction of virtual chromosomes of manufactured products for their gene-engineering-based innovative design," Computer-Aided Design, vol. 37, no. 11, pp. 1191-1203, 2005.

[29] H.-C. Tsai and J.-R. Chou, "Automatic design support and image evaluation of two-coloured products using colour association and colour harmony scales and genetic algorithm," Computer-Aided Design, vol. 39, no. 9, pp. 818-828, 2007.
[30] S.-W. Hsiao, C.-F. Hsu, and K.-W. Tang, "A consultation and simulation system for product color planning based on interactive genetic algorithms," Color Research \& Application, vol. 38, no. 5, pp. 375-390, 2013.

[31] Y. Sun, W.-l. Wang, Y.-w. Zhao, and X.-j. Liu, "User image oriented interactive genetic algorithm evaluation mode in product development," Computer Integrated Manufacturing Systems, vol. 18, no. 2, pp. 276-281, 2012.

[32] R. Kamalian, E. Yeh, Z. Ying, A. M. Agogino, and H. Takagi, "Reducing human fatigue in interactive evolutionary computation through fuzzy systems and machine learning systems," in Proceedings of the 2006 IEEE International Conference on Fuzzy Systems, Vancouver, Canada, July 2006.

[33] Z. Gu, M. Xi Tang, and J. H. Frazer, "Capturing aesthetic intention during interactive evolution," Computer-Aided Design, vol. 38, no. 3, pp. 224-237, 2006.

[34] G. Harih, M. Borovinšek, and Z. Ren, Optimisation of Product's Hand-Handle Interface Material Parameters for Improved Ergonomics, Springer International Publishing, Cham, Switzerland, 2015.

[35] P. Cheng, D. Chen, and J. Wang, "Clustering of the body shape of the adult male by using principal component analysis and genetic algorithm-BP neural network," Soft Computing, vol. 24, no. 17, Article ID 13219, 2020.

[36] N. M. B. Serrano, P. J. G. Nieto, A. S. Sánchez, F. S. Lasheras, and P. R. Fernández, A Hybrid Algorithm for the Assessment of the Influence of Risk Factors in the Development of Upper Limb Musculoskeletal Disorders, Springer, Cham, Switzerland, 2018.

[37] S. S. Sankar, O.-M. Holman, A. F. Gazabon, and C. J. Acevedo, "Application of genetic algorithm to job scheduling under ergonomic constraints in manufacturing industry," Journal of Ambient Intelligence and Humanized Computing, vol. 10, no. 5, pp. 2063-2090, 2019.

[38] A.-Z. Atiya, L. Lee, and K. Xing, "Developing a multi-objective genetic optimisation approach for an operational design of a manual mixed-model assembly line with walking workers," Journal of Intelligent Manufacturing, vol. 27, no. 5, pp. 1-17, 2014.

[39] P. Jedlicka and T. Ryba, "Genetic algorithm application in image segmentation," Pattern Recognition and Image Analysis, vol. 26, no. 3, pp. 497-501, 2016.

[40] L. V. Campenhout, J. Frens, K. Overbeeke, A. Standaert, and H. Peremans, "Physical interaction in a dematerialized world," International Journal of Design, vol. 7, no. 1, pp. 1-18, 2013.

[41] K. Nandhini and S. R. Balasundaram, "Improving readability through individualized summary extraction, using interactive genetic algorithm," Applied Artificial Intelligence, vol. 30, no. 7, pp. 635-661, 2016.

[42] R. M. Gray, Entropy and Information Theory, Springer, New York, NY, USA, 2nd edition, 2011.

[43] B. Anderhofstadt and S. Spinler, "Factors affecting the purchasing decision and operation of alternative fuel-powered heavy-duty trucks in Germany-a Delphi study," Transportation Research Part D: Transport and Environment, vol. 73, pp. 87-107, 2019.

[44] X.-m. Wu and Y. Feng, "Reactive power optimization of distribution network based on improved genetic algorithm," Journal of Xi'an Shiyou University (Natural Science Edition), vol. 30, no. 3, pp. 95-99, 2015.

[45] Z. Ling and M.-j. Li, "State space evolutionary algorithm based on non-uniform mutation operator," Computer Technology and Development, vol. 28, no. 9, pp. 68-71, 2018. 
[46] W. Hu and J. Zhao, "Automobile styling gene evolution driven by users' expectation image," Journal of Mechanical Engineering, vol. 47, no. 16, pp. 176-181, 2011.

[47] J. J. Scott and R. Gray, "A comparison of tactile, visual, and auditory warnings for rear-end collision prevention in simulated driving," Human Factors: The Journal of the Human Factors and Ergonomics Society, vol. 50, no. 2, pp. 264-275, 2008.

[48] B. Reimer, B. Mehler, and J. F. Coughlin, "Reductions in selfreported stress and anticipatory heart rate with the use of a semi-automated parallel parking system," Applied Ergonomics, vol. 52, pp. 120-127, 2016.

[49] B. D'Ippolito, “The importance of design for firms? competitiveness: a review of the literature," Technovation, vol. 34, no. 11, pp. 716-730, 2014.

[50] S. Asensio-Cuesta, J. A. Diego-Mas, L. Canós-Darós, and C. Andrés-Romano, "A genetic algorithm for the design of job rotation schedules considering ergonomic and competence criteria," International Journal of Advanced Manufacturing Technology, vol. 60, no. 9-12, pp. 1161-1174, 2012.

[51] S.-C. Lee, H.-E. Tseng, C.-C. Chang, and Y.-M. Huang, "Applying interactive genetic algorithms to disassembly sequence planning," International Journal of Precision Engineering and Manufacturing, vol. 21, no. 4, pp. 663-679, 2020.

[52] M. G. Shishaev, V. V. Dikovitsky, and L. V. Lapochkina, "The experience of building cognitive user interfaces of multidomain information systems based on the mental model of users," in Proceedings of the 6th Computer Science On-Line Conference (CSOC 2017), Prague, Czech Republic, April 2017.

[53] S. Lee, H. Alzoubi, and S. Kim, "The effect of interior design elements and lighting layouts on prospective occupants' perceptions of amenity and efficiency in living rooms," Sustainability, vol. 9, no. 7, Article ID 1119, 2017.

[54] G. Caruso, "Mixed reality system for ergonomic assessment of driver's seat," International Journal of Virtual Reality, vol. 10, no. 2, pp. 69-79, 2011.

[55] M. L. Reyes and J. D. Lee, "Effects of cognitive load presence and duration on driver eye movements and event detection performance," Transportation Research Part F: Traffic Psychology and Behaviour, vol. 11, no. 6, pp. 391-402, 2008.

[56] D. Cisler, P. M. Greenwood, D. M. Roberts, R. McKendrick, and C. L. Baldwin, "Comparing the relative strengths of EEG and low-cost physiological devices in modeling attention allocation in semiautonomous vehicles," Frontiers in Human Neuroscience, vol. 13, Article ID 109, 2019.

[57] I.-H. Kim, J.-W. Kim, S. Haufe, and S.-W. Lee, "Detection of braking intention in diverse situations during simulated driving based on EEG feature combination," Journal of Neural Engineering, vol. 12, no. 1, Article ID 016001, 2014. 